\title{
ARTIGOS
}

\section{MORTE SÚBITA EM ÁREA ENDÊMICA DA DOENÇA DE CHAGAS}

\author{
Edison Reis Lopes e Edmundo Chapadeiro
}

\begin{abstract}
Em área endêmica da doença de Chagas foi realizado estudo da freqüência e da etiologia da morte súbita. Constatou-se que a proporçâo destes casos na área estudada equipara-se à observada em outros centros do pais, onde estudos similares foram feitos; entretanto é relativamente baixa quando comparada com a de outros países. Quanto às causas da subitaneidade do óbito, verificou-se que a etiologia mais freqüente é representada pelas doenças cardiovasculares, sendo a doença de Chagas a principal responsável em individuos de mais de 15 anos de idade.
\end{abstract}

Palavras chaves: Doença de Chagas. Tripanossomíase. Morte súbita. Cardiopatia chagástica.

A análise da vasta literatura referente à doença de Chagas revela a importância que se dá à morte súbita nesta endemia. O próprio Carlos Chagas ${ }^{4}$ mencionava que na localidade de Lassance (Minas Gerais), não existia família que não tivesse perdido um ou vários membros vitimados pela morte súbita e inesperada. Koberle ${ }^{11}$ relata que $30 \%$ dos chagásicos necropsiados em Ribeirão Preto faleceram subitamente, sendo a morte determinada por fibrilação ventricular. Baseado em dados clínicos, Porto $^{22}$ afirma que, nas zonas endêmicas da doença de Chagas, a tripanossomíase é a causa mais frequiente de óbito súbito e que este ocorreu em 37,5\% dos chagásicos por ele estudados. Realizando estudos de campo, na área endêmica de São Felipe (Bahia), Macêdo ${ }^{20}$ observou uma taxa de letalidade devida à doença de Chagas de $2,8 \%$ e em $37,5 \%$ dos chagásicos por ela estudados, a morte foi súbita. Lopes e cols ${ }^{18}$, estudando de modo sistematizado, em material de necropsias, o tipo de morte do chagásico crônico, observaram $9,8 \%$ de óbitos súbitos, em conseqüência da doença.

A leitura dos trabalhos citados revela, todavia, que o critério de morte súbita em doença de Chagas é vago e controvertido. Alguns dos trabalhos citados e outros mais 514151723 são referentes a casos de tripanossomóticos falecidos de modo repentino, os quais não apresentaram, tanto quanto se pôde apurar, sinais e/ou sintomas da enfermidade. Outros, entretanto ${ }^{6} 72021222425$, englobam em sua

\footnotetext{
Trabalho do Departamento de Patologia e Medicina Legal da Faculdade de Medicina do Triângulo Mineiro, realizado com auxílio do Conselho Nacional de Desenvolvimento Científico e Tecnológico do Brasil.

Endereço para correspondência: Faculdade de Medicina do Triângulo Mineiro, 38.100 - Uberaba - MG - Brasil.

Recebido para publicação em $3 / 5 / 83$
}

casuistica também chagásicos falecidos repentinamente, nos quais porém o óbito foi precedido de manifestações da doença.

De acordo com os conceitos existentes na literatura sobre a morte súbita em geral (ver Lopes ${ }^{16}$ ), consideramos como morte súbita em doença de Chagas aqueles casos de chagásicos falecidos repentinamente que não apresentaram, tanto quanto se pôde apurar, manifestações clínicas da tripanossomiase cruzi e nos quais o tempo decorrido entre o início dos sintomas e/ou sinais e o evento letal não tenha ultrapassado 24 horas.

Não há, ao que nos consta, na literatura estudos sistematizados com relação à prevalência deste tipo de óbito do chagásico no panorama geral das mortes súbitas. Pelo que pudemos constatar, somente Rocha (A Rocha: comunicação pessoal, 1974) realizou levantamento sobre a ocorrência da morte súbita em 394 necropsias em Uberlândia, no Triângulo Mineiro, onde a doença de Chagas éendêmica. Entretanto, o referido autor não publicou șeus resultados.

Diante dos fatos expostos e associando-os à observação popular de que a subitaneidade do óbito no Triângulo Mineiro é bastante freqüente, resolvemos estudar a real frequiência e a etiologia da morte súbita em Uberaba.

\section{MATERIAL E METODOS}

O material de estudo foi obtido em Uberaba, cidade situada no Triângulo Mineiro, que à época da realização deste trabalho contava com uma população (rural e urbana) de cerca de 200.000 habitantes. Para análise da frequiência e da etiologia das mortes súbitas duas fontes de informação fo- 
ram utilizadas. 1ํ) - Cartório de Registro Civil, onde analisamos as 1.618 certidões de óbitos relativas a todos os falecimentos ocorridos no municipio de Uberaba, em 1980; $2^{\circ}$ ) - Necropsias realizadas na Faculdade de Medicina do Triângulo Mineiro no período de 1971-1980, em um total de 1.454 óbitos.

Tendo em vista que uma das finalidades primordiais de nosso estudo é a realização de análises comparativas com os dados de outros autores, especialmente com os de Laurenti e cols ${ }^{13}$, utilizamos somente os casos de óbitos ocorridos em pessoas maiores de 15 anos de idade.

No exame das certidões de óbitos, abandonamos também as que nào se enquadravam no conceito de morte súbita por nós utilizado. Assim, eliminamos preliminarmente todos os casos relativos a mortes violentas, neoplasias, doenças caquetizantes etc. Nos restantes, através de entrevista com familiares, médicos, etc., pudemos determinar, de modo bastante seguro, os verdadeiros casos de morte súbita. Da mesma forma, através dos protocolos das necropsias realizadas nos serviços de Medicina Legal e Patologia da Faculdade de Medicina do Triângulo Mineiro, foram selecionados os casos de morte súbita. Além dos exames morfológicos das visceras, efetuamos em todos os casos as reações sorológicas para doença de Chagas no líquido pericárdico. Quando necessário, foram feitas outras análises (toxicológicas, etc.)

\section{RESULTADOS}

Das 1.618 certidões de óbito registradas no Cartório de Registro Civil de Uberaba em 1980, 910 , ou seja, $56,3 \%$ eram de homens, $704(43,5 \%)$ de mulheres e em $4(0,2 \%)$ não constava o sexo. Deste total, 1.226 certidões referiam-se a pessoas maiores de 15 anos de idade, das quais $688(56,1 \%)$ eram do sexo masculino, $536(43,7 \%)$ do sexo feminino e $2(0,2 \%)$ não tinham indicação de sexo.

Desses 1.226 adultos, 976 tiveram morte natural, sendo $547(56 \%)$ homens, $425(43,5 \%)$ mulheres e $4(0,5 \%)$ não tinham o sexo referido.

Por outro lado, dessas 976 certidões de maiores de 15 anos de idade, em 54 (5,5\%) houve morte súbita, em $911(93,4 \%)$ não-súbita, e em 11 casos $(1,1 \%)$ não pudemos determinar a subitaneidade ou não da morte. Dos 54 casos de morte súbita, 37 $(68,5 \%)$ ocorreram no sexo masculino e $17(31,5 \%)$ no feminino.

A distribuição destes 54 casos de morte súbita, segundo a etiologia do óbito, encontra-se na Tabela 1.

Tabela 1 - Causas de mortes súbitas em Uberaba no ano de 1980

\begin{tabular}{|c|c|c|c|}
\hline Causa Mortis & $\begin{array}{l}N^{o} \text { de } \\
\text { casos }\end{array}$ & $\begin{array}{l}\% d o \\
\text { total }\end{array}$ & $\begin{array}{c}\text { \%dos } \\
\text { parciais }\end{array}$ \\
\hline $\begin{array}{l}\text { Sistema Cardiovascular } \\
\text { (coração aorta) }\end{array}$ & 28 & 51,9 & - \\
\hline $\begin{array}{l}\text { Cardite Chagásica Crônica } \\
\text { (C. Ch. Cr.) }\end{array}$ & 13 & 24,1 & 46,4 \\
\hline $\begin{array}{l}\text { Cardiopatia isquêmica } \\
\text { (Coronariana) }\end{array}$ & 12 & 22,2 & 42,8 \\
\hline C. Ch. Cr. + cardiopatia isquêmica & 2 & 3,7 & 7,2 \\
\hline Aneurisma da aorta abdominal & 1 & 1,9 & 3,6 \\
\hline Sistema Respiratório & 9 & 16,6 & - \\
\hline Edema pulmonar agudo & 5 & 9,3 & 55,5 \\
\hline Pneumonia & 2 & 3,6 & 22,3 \\
\hline Embolia pulmonar & 1 & 1,9 & 11,1 \\
\hline Edema das pregas aritenoepiglóticas & 1 & 1,9 & 11,1 \\
\hline Sistema Nervoso & 5 & 9,3 & - \\
\hline Aneurisma cerebral roto & 2 & 3,6 & 40,0 \\
\hline Hemorragia intracerebral & 1 & 1,9 & 20,0 \\
\hline Embolia cerebral & 1 & 1,9 & 20,0 \\
\hline Meningite & 1 & 1,9 & 20,0 \\
\hline Sistema Digestivo & 1 & 1,9 & - \\
\hline Pancreatite aguda & 1 & 1,9 & 100 \\
\hline Indeterminada & 11 & 20,3 & - \\
\hline Total & 54 & - & - \\
\hline
\end{tabular}

Observações: Fonte: Certidòes de óbitos registradas no Cartório de Registro Civil. Total de óbitos registrados no ano: 1.618. 
Lopes ER. Chapadeiro E. Morte súbita em área endêmica da doença de Chagas. Revista da Sociedade Brasileira de Medicina Tropical 16:79-84, Abr-Jun, 1983

Tabela 2 - Causas de morte súbita em 1.236 necropsias de adultos realizadas na Faculdade de Medicina ao Trángulo Mineiro no periodo de $1971-1980$

\begin{tabular}{|c|c|c|c|}
\hline Causa Mortis & $\begin{array}{l}\text { No de } \\
\text { Casos }\end{array}$ & $\begin{array}{l}\% \text { do } \\
\text { Total }\end{array}$ & $\begin{array}{l}\text { \%dos } \\
\text { parciais }\end{array}$ \\
\hline $\begin{array}{l}\text { Sistema cardiovascular } \\
\text { (coração aorta) }\end{array}$ & 79 & 79 & - \\
\hline $\begin{array}{l}\text { Cardite Chagásica Crônica } \\
\text { (C. Ch. Cr.) }\end{array}$ & 62 & 62 & 78,5 \\
\hline Cardiopatia isquêmica (coronariana) & 10 & 10 & 12,5 \\
\hline Cardiopatia hipertensiva + coronariana & 3 & 3 & 3,8 \\
\hline C. Ch. Cr. + Cardite reumática & 1 & 1 & 1,3 \\
\hline C. Ch. Cr. + Cardiopatia hipertensiva & 1 & 1 & 1,3 \\
\hline Aneurisma dissecante da aorta & 2 & 2 & 2,6 \\
\hline Sistema Nervoso & 9 & 9 & - \\
\hline Aneurisma cerebral roto & 5 & 5 & 55,6 \\
\hline Hemorragia intracerebral & 3 & 3 & 33,3 \\
\hline Meningite & 1 & 1 & 11,1 \\
\hline Sistema Respiratório & 4 & 4 & - \\
\hline Pneumonia & 3 & 3 & 75,0 \\
\hline Edema agudo dos pulmões & 1 & 1 & 25,0 \\
\hline Sistema Digestivo & 2 & 2 & - \\
\hline Úlcera péptica perfurada & 1 & 1 & 50,0 \\
\hline Pancreatite aguda necro-hemorrágica & 1 & 1 & 50,0 \\
\hline Outros & 1 & 1 & - \\
\hline Coagulação intravascular disseminada & 1 & 1 & 100 \\
\hline Indeterminada & 5 & 5 & - \\
\hline Total & 100 & 100 & - \\
\hline
\end{tabular}

Nosso outro material de estudo baseou-se em dados de necropsias. No período de 1971 a 1980 , realizaram-se na Faculdade de Medicina do Triângulo Mineiro 1.454 autópsias, das quais $1.236 \mathrm{em}$ maiores de 15 anos de idade. Dessas 1.236 autópsias de adultos que tiveram morte natural, em 100 $(8,1 \%)$ se pode caracterizar que houve morte súbita. A Tabela 2 indica a distribuição desses 100 óbitos súbitos segundo a etiologia da morte.

\section{DISCUSSÃO}

A análise de nossos resultados, que revelaram $5,5 \%$ de mortes súbitas registradas no Cartório de Registro Civil de Uberaba em 1980 e $8,1 \%$ nas necropsias realizadas na Faculdade de Medicina no periodo de 1971 a 1980 , mostra que a proporção dos casos de morte súbita em nossa região é relativamente baixa, quando comparada com a relatada pelos autores estrangeiros. Assim, Burch e cols ${ }^{2}$ afirmam que 15 a $30 \%$ dos óbitos naturais nos EUA ocorrem subitamente. Canger Rodrigues ${ }^{3}$, citando trabalhos estrangeiros, menciona a incidência aproximada das mortes súbitas em torno de $10 \%$. Kuller ${ }^{2}$, no grupo etário de 40 a 64 anos, observou $32 \%$ das mortes ocorrendo subitamente. Nossos achados referentes ao Cartório de Registro Civil de Uberaba estão bem próximos dos observados por Laurenti e cols ${ }^{13}$, em estudo similar realizado na cidade de São Paulo. Esses autores observaram que $5,82 \%$ das mortes naturais foram do tipo súbito e inesperado. A metodologia que Laurenti e cols ${ }^{13}$ empregaram foi muito semelhante à que utilizamos. Nossos dados de necropsias se aproximam bastante dos encontrados por Rocha (A Rocha: comunicação pessoal, 1974) em Uberlândia, que constatou, também em necropsias, a morte súbita em $9,4 \%$ dos óbitos naturais ocorridos em adultos. 
As discrepâncias entre nossos resultados e os de alguns outros autores podem ser explicadas por vários fatores. Primeiro, pelas diferenças conceituais de morte súbita; segundo, porque a exemplo de Laurenti e cols ${ }^{13}$, procuramos ser rigorosos na caracterização do tipo de óbito. Alguns de nossos casos, inicialmente considerados como morte súbita, foram excluídos do presente estudo, após entrevista com familiares, quando se apurou que o falecido apresentara, antes do óbito, manifestações da doença; terceiro, porque não incluímos em nossa casuística menores de 15 anos de idade; quarto, porque existem realmente diferenças regionais como, por exemplo, a elevada frequiência em nossa casuística de mortes súbitas devido à doença de Chagas.

Outro aspecto a discutir é o da diferença entre as freqüências de morte súbita por nós obtidas nas certidões de óbitos e nas necropsias. O material de necropsias não constitui boa amostra da população, pois geralmente elas são feitas nas classes sócio-econômicas menos favorecidas. Ora, como sabemos, há doenças, como a cardiopática isquêmica, que freqüentemente causam morte súbita e que incidem com maior intensidade nos indivíduos das classes sócio-econômicas mais elevadas. Por este e outros motivos cremos que os nossos dados obtidos a partir das certidões de óbitos são mais representativos da população do que os obtidos em material de necropsias.

Quanto à frequiência da morte súbita em relação ao sexo, os dados obtidos em nossas duas amostragens populacionais mostraram que aquela ocorreu mais nos homens do que nas mulheres, o que concorda com os dados da literatura. ${ }^{13}$

Concordando com os dados da literatura, nossos resultados demonstram que a etiologia mais freqüente de morte súbita é representada pelas doenças cardiovasculares ${ }^{38} 91012131926$. Entretanto, ao contrário da maioria desses autores, em nossa casuística, a doença de Chagas (provavelmente através de cardite chagástica crônica) foi a principal causa de morte súbita. Em $13(24,1 \%)$ das mortes súbitas por nós detectadas nas certidões de óbito, e em $62 \%$ das necropsias, o evento letal foi devido a esta causa. $O$ valor mais alto observado nos casos provenientes das necropsias deve-se provavelmente ao maior rigor do diagnóstico que a autópsia confere. A causa mortis não pôde ser determinada em 11 mortes súbitas (Tabela 1). $\overline{\mathrm{E}}$ bastante provável que a maior percentagem desses casos fosse de doença de Chagas. Não se pode afastar a tripanossomíase cruzi como responsável pelo óbito súbito, nos dois casos em que apareceu associada à cardiopatia isquêmica. De modo similar, nas necropsias também haviam casos de associações de cardite chagástica com cardite reumática e com cardiopatia hipertensiva.

A doença de Chagas é a principal causa de morte súbita em nosso meio, em indivíduos de mais de 15 anos, o que concorda com as observações de Rocha (A Rocha: comunicação pessoal, 1974). Porto ${ }^{22}$ já afirmava em 1963 que, nạs zonas endêmicas de doença de Chagas, ela é a causa mais comum de morte súbita, ultrapassando, de muito, a arterosclerose das coronárias. Ainda que o critério conceitual de morte súbita empregado por Porto ${ }^{22}$ não seja idêntico ao que utilizamos, sua afirmação é bastante significativa.

A segunda causa de morte súbita, em nossa casuistica, foi a cardiopatia isquêmica. A percentagem de sua frequiência em nosso material (aproximadamente $22 \%$ nas certidões de óbito e $10 \%$ nas necropsias), está abaixo da relatada por Laurenti e cols $^{13}$ e Canger Rodrigues ${ }^{3}$ em São Paulo, que a observaram em aproximadamente $40 \%$ de seus casos. Nossa casuística quanto à cardiopatia isquêmica também está muito abaixo da referida por autores estrangeiros ${ }^{9} 1226$.

A explicação para essas discrepâncias talvez possa ser a procedência de nossa amostra populacional, em sua maior parte de zona rural e oriunda de cidade de pequeno e médio portes. Sabe-se que mais de $90 \%$ das cardiopatias isquêmicas ou coronarianas são devidas a arterosclerose ${ }^{1}$, mais freqüente na população urbana e das grandes cidades. Portanto é de se esperar que as mortes súbitas por cardiopatia isquêmica sejam mais frequientes neste último grupo populacional.

Os aneurismas aórticos e outras doenças cardiovasculares, a exemplo do relato na literatura, constituíram, também, em nosso material, causa bem menos freqüente de mortes súbitas.

Em nossa casuística, provenientes das certidões de óbitos registradas no Cartório de Registro Civil de Uberaba em 1980, as doenças respiratórias ocupam o segundo lugar como causa de morte súbita; mas na casuística baseada nos dados necroscópicos, o sistema respiratório está em terceiro plano. $O$ inverso sucede com as enfermidades do sistema nervoso, que na primeira casuística estão em terceiro lugar, e na outra, em segundo. Cremos que o pequeno número de casos que forneceu uma 
Lopes ER, Chapadeiro E. Morte súbita em área endêmica da doença de Chagas. Revista da Sociedade Brasileira de Medicina Tropical 16:79-84, Abr-Jun, 1983

amostragem reduzida, especialmente na primeira casuística, seja a responsável por essa discrepância. Talvez pelo pequeno número de óbitos súbitos causados por doenças desses sistemas, seja difícil saber sua real frequiencia neste tipo de morte. Das doenças do sistema nervoso, a principal etiologia de morte súbita, em nossa casuística, foram os aneurismas cerebrais e, das respiratórias, o edema agudo dos pulmões e as pneumonites. Estes achados, de um modo geral, concordam com os relatos na literatura ${ }^{19}$. Outras condições causadoras de morte súbita são raras, como demonstram nosso material e os relatos da literatura.

\section{$S U M M A R Y$}

A study of the frequency and etiology of sudden death in an area with endemic Chagas disease is reported. The proportion of cases is about the same as elsewhere in this country, where equivalent studies have been done. It is relatively low when compared with that of some other countries. Cardiovascular disease is the most frequent cause of sudden death in most countries. In our study chronic chagasic cardiomyopathy was the major cause of sudden death in people over 15 years of age.

Key words: Chagas disease, American trypanosomiasis. Sudden death. Chagasic cardiomyopathy.

\section{AGRADECIMENTOS}

Agradecemos a Sra. Iolanda Barboza de Araújo Borges pela assistência na parte datilográfica e ao Sr. José Antônio Alves Filho pelo auxílio na parte técnica.

\section{REFERENCIAS BIBLIOGRAFICAS}

1. Bogliolo L. Miocárdio. Endocárdio. Pericárdio. In: Bogliolo L, Patologia. 3! Ed. Guanabara - Koogan, Rio de Janeiro, p. 342-395, 1981.

2. Burch GE, De Pasquale NP. Sudden unexpected natural death. American Journal of Medical Science 112: 86-123, 1965.

3. Canger Rodrigues A. Morte súbita e morte suspeita em medicina legal. Revista da Associação Médica Brasileira 19:203-214, 1973.

4. Chagas C. O mal de Chagas. Archivos da Sociedade de Medicina e Cirurgia de São Paulo 3:24-66, 1912.
5. Chapadeiro E, Lopes ER, Raso P, Brauna AO. Importância do reconhecimento da doença de Chagas em medicina legal. O Hospital 76:249-257, 1969.

6. Couto Filho M. Lesões do sistema específico do miocárdio num caso de tripanossomíase americana. O Hospital 8:1223-1227, 1936.

7. Dias JC. A cardiopatia crônica na moléstia de Chagas. Revista Clínica e Científica 19:9-17, 1950.

8. Goldstein S. Sudden death and coronary heart disease. Futura Publishing Co. Inc. Mount Kisco, New York, 1974.

9. Helpern M, Rabson SM. Sudden and unexpected natural death. General considerations and statistics. New York Journal of Medicine 45:1197-1201, 1945.

10. Janes LR. Common causes of unexpected deaths. In: Gradwohz RBH. Legal Medicine, C.V.Mosby, St. Louis, p. 343-393, 1954.

11. Koberle F. Cardiopatia chagásica. O Hospital 53:311-346, 1958.

12. Kuller L. Sudden and unexpected non traumatic deaths in adults: a review of epidemiological and clinical studies. Journal of Chronical Diseases 19:1165$1192,1966$.

13. Laurenti R, Pacheco de Souza JM, Melo Prado MH, Gotlieb SC. Estudo epidemiológico da morte súbita em adultos de 15 a 74 anos na cidade de São Paulo. Arquivos Brasileiros de Cardiologia 35:5-14, 1980.

14. Lopes ER. Contribuição ao estudo dos gânglios cardíacos (sistema nervoso autônomo) em chagásicos crônicos. Tese de doutorado. Faculdade de Medicina do Triângulo Mineiro, Uberaba, Minas Gerais, 1965.

15. Lopes ER. Estudo comparativo dos gânglios subepicárdicos nas cardiopatias chagásicas crônicas, reumáticas e hipertensiva. Tese de livre-docência. Faculdade de Medicina do Triângulo Mineiro, Uberaba, Minas Gerais, 1969.

16. Lopes ER. Morte súbita em área endêmica da doença de Chagas. Sua importância médico-legal. Tese de Professor Titular. Faculdade de Medicina do Triângulo Mineiro, Uberaba, Minas Gerais, 1981 .

17. Lopes ER, Chapadeiro E, Almeida HO, Rocha A. Contribuição ao estudo da anatomia patológica dos corações de chagásicos falecidos subitamente. Revista da Sociedade Brasileira de Medicina Tropical 9:269-282, 1975.

18. Lopes ER, Guimarães AHB, Rocha A. Tipos de morte no chagásico crônico. In: Resumos do XIX Congresso da Sociedade Brasileira de Medicina Tropical, Rio de Janeiro, p. 23, 1983.

19. Luke JL, Helpern M. Sudden unexpected death from natural causes in young adults. A review of 275 consecutive autopsied cases. Archives of Pathology $85: 10-17,1968$ 
Lopes ER, Chapadeiro E. Morte súbita em área endêmica da doença de Chagas. Revista da Sociedade Brasileira de Medicina Tropical 16:79-84, Abr-Jun, 1983

20. Macêdo VO. Influência da exposição e reinfecção na evolução da doença de Chagas. Estudo longitudinal de 5 anos. Tese de livre-docência. Faculdade de Medicina da Universidade Federal do Rio de Janeiro, 1973.

21. Menezes M. Miocardite chagásica crônica: sua incidência no Brasil Central. O Hospital 24:167-179, 1949.

22. Porto CC. Contribuição do eletrocardiograma no prognóstico e evolução da doença de Chagas. Tese de doutoramento. Faculdade de Medicina da Universidade Federal de Minas Gerais, Belo Horizonte, 1963.
23. Porto CC. A doença de Chagas do ponto de vista médico-trabalhista. Arquivos Brasileiros de Cardiologia 27:85-91, 1974.

24. Prata A. Prognóstico e complicações da doença de Chagas. Revista Goiana de Medicina 5:87-96, 1959.

25. Rassi A, Carneiro O. Estudo clínico, eletrocardiográfico e radiológico da cardiopatia chagásica crônica. Análise de 106 casos. Revista Goiana de Medicina 2:287-296, 1956.

26. Spain D, Braders VA, Mohr C. Coronary artherosclerosis as a cause of unexpected and unexplained death. An autopsy study from 1949-1959. The Journal of the American Medical Association 174: 384$388,1960$. 